\title{
HOMOTOPY K3 SURFACES AND MOD 2 SEIBERG-WITTEN INVARIANTS
}

\author{
John W. Morgan And Zoltán Szabó
}

\section{Introduction}

The purpose of this note is to establish the following result.

Theorem 1.1. Let $X$ be a closed smooth four-manifold which is homotopy equivalent to a K3 surface. Let $P \rightarrow X$ be the unique Spin ${ }^{c}$-structure up to isomorphism with trivial determinant line bundle. Then the value of the Seiberg-Witten invariant of $P$ is congruent to one modulo two.

After writing this paper we learned that this result was also known to Kronheimer and Furuta.

This theorem is closely related to the work of Fintushel and Stern [FS1], [FS2]. They proved that any homotopy $K 3$ surface which contains a smoothly embedded $\Sigma(2,3,7)$ has nontrivial $S U(2)$ Donaldson invariants mod 2. Note that Theorem 1.1 and the folk conjecture, (cf. [W]), on the relation between Donaldson and Seiberg-Witten invariants would imply that every homotopy $K 3$ surface has nontrivial $S U(2)$ Donaldson invariants mod 2.

As a straightforward corollary of Theorem 1.1 and the adjunction inequalities for the Seiberg-Witten basic classes [KM], [MSzT], [D2] we get the following:

Corollary 1.2. Let $X$ be a closed smooth four-manifold which is homotopy equivalent to the $K 3$ surface. For every $x \in H_{2}(X, \mathbb{Z})$, let $g(x)$ denote the minimal genus of smoothly embedded riemannian surfaces representing $x$. Then for each $x \neq 0$ with $x \cdot x \geq 0$ we have

$$
2 g(x)-2 \geq x \cdot x
$$

The proof of Theorem 1.1 follows closely the lines of argument laid down by Kronheimer when he proves using Seiberg-Witten invariants that homotopy $K 3$ surfaces are indecomposable.

Received May 3, 1996. 


\section{Proof of Theorem 1.1}

Fix $X$ and $P$ as in the statement. Fix a riemannian metric $g$ on $X$. First notice that the formal dimension of the moduli space associated to the Seiberg-Witten equations for this $\operatorname{Spin}^{c}$-structure is zero. Recall that the plus spin bundle $S^{+}(P) \rightarrow X$ associated to $P$ is determined by the plus spin representation of the Clifford algebra $\Delta^{+}: C l_{0}\left(\mathbb{R}^{4}\right) \rightarrow \operatorname{End}\left(\mathbb{C}^{2}\right)$. We view $\mathbb{C}^{2}$ as the quaternions $\mathbb{H}$ under the identification $(\alpha, \beta) \mapsto \alpha+j \beta$. Then the representation of $C l_{0}\left(\mathbb{R}^{4}\right)$ is by left quaternionic multiplication so that it commutes with the right multiplication by quaternions. In particular, we have an action of the subgroup $J$ of unit quaternions generated by $j$ and the unit circle in the complex numbers on $\mathbb{C}^{2}$ commuting with $\Delta^{+}$. In terms of complex coordinates the action of $j$ is given by

$$
(\alpha, \beta) \mapsto(-\bar{\beta}, \bar{\alpha})
$$

This in turn determines an action of the group $J$ on the space of sections $\Gamma\left(S^{+}(P)\right)$. (Here we use that fact that the determinant line bundle of $P$ is trivial, and we also fix a riemannian metric on $X$ ) There is a completely analogous action on $\mathbb{C}^{2}$ commuting with the representation of $\Delta^{-}$, and hence an induced action of the group $J$ on $\Gamma\left(S^{-}(P)\right)$. The Dirac operator and Clifford multiplication commute with these actions of $J$. We also let $J$ act on the spaces of differential forms by having the unit circle act trivially and the element $j$ act by multiplication by -1 . Lastly, we define an action of $J$ on the group of gauge transformations by letting the unit circle act trivially and letting $j$ act by complex conjugation. In this way we define actions of $J$ on the space of configurations $\mathcal{C}$, on the value space for the Seiberg-Witten functional, and on the gauge group. The gauge action and the Seiberg-Witten equations commute with the $J$ actions.

In particular, there is an induced action of $J$ on the space $\mathcal{B}^{0}$ of based configurations mod gauge which leaves invariant the based moduli space of solutions to the Seiberg-Witten equations for $P$. The action of the circle subgroup of $J$ is just the usual action of $S^{1}$ producing as quotient spaces, the space $\mathcal{B}$ of configurations and the moduli space $\mathcal{M}$ of solutions. Notice that in the moduli space there is exactly one reducible solution - the product connection on the determinant line bundle and the trivial spinor field. From now on we denote this solution by $\theta_{0}$.

Our goal is to perturb the Seiberg-Witten equations, leaving them $J$ invariant until the reducible solution, which must be a solution by $J$-invariance, is isolated and is given by the same local model as in the case of the $K 3$ surface. First notice that the (complex) index of the Dirac operator associated with the trivial connection on the determinant line bundle of $P, \not \partial: \Gamma\left(S^{+}(P)\right) \rightarrow \Gamma\left(S^{-}(P)\right)$ is two. Note also that $J$ has a semi-free action on Ker $\not \partial$, Coker $\not \partial$. The local model of the moduli space around $\theta_{0}$ is determined by the Kuranishi map:

$$
Q: \operatorname{Ker} \not \partial \rightarrow \operatorname{Coker} \not \supset \oplus \mathcal{H}_{+}^{2}(X ; i \mathbb{R})
$$


where $\mathcal{H}_{+}^{2}(X ; i \mathbb{R}) \cong \mathbb{R}^{3}$ denotes the space of self-dual harmonic purely imaginary two-forms on $X$.

Unfortunately, at this point we have no control over the Kuranishi map and consequently no control over whether the reducible solution is isolated in the based moduli space. Now we perturb the equations in order to gain the requisite control over the local model at the reducible solution.

Notice that the $J$ actions on Ker $\not \partial$ and Coker $\not \partial$ give these spaces the structure of a quaternionic vector space. Let $P: \operatorname{Ker} \not \partial \rightarrow$ Coker $\not \partial$ be a quaternionic linear surjection. Note that $P$ commutes with the $J$ actions. Then $\operatorname{Ker} P \cong \mathbb{H}$ and the leading term of

$$
\left.Q\right|_{\operatorname{Ker} P}: \operatorname{Ker} P \rightarrow \mathcal{H}_{+}^{2}(X ; i \mathbb{R})
$$

is a quadratic polynomial map which is $J$ invariant. We denote this quadratic map by $Q_{0}$. The space Quad of $J$-invariant quadratic polynomial maps from $\mathbb{H}$ to $\mathbb{R}$ is three dimensional (isomorphic to $\mathbb{R}^{3}$ ) and a basis for these maps is

$$
\begin{aligned}
\left(z_{1}, z_{2}\right) & \mapsto\left|z_{1}\right|^{2}-\left|z_{2}\right|^{2}, \\
\left(z_{1}, z_{2}\right) & \mapsto \operatorname{Re}\left(z_{1} \bar{z}_{2}\right) \\
\left(z_{1}, z_{2}\right) & \mapsto \operatorname{Im}\left(z_{1} \bar{z}_{2}\right) .
\end{aligned}
$$

We can view $Q_{0}$ as a linear map from the dual space to $H_{+}^{2}(X ; i \mathbb{R})$ to Quad. We want this map to be an isomorphism, but in fact it can be arbitrary. We fix $R: \operatorname{Ker} P \rightarrow \mathcal{H}_{+}^{2}(X ; i \mathbb{R})$ to be a quadratic form whose associated linear map is an isomorphism. Then for almost all $t \in \mathbb{R}$ we have that $Q_{0}+t R$ is a quadratic form whose associated linear map is an isomorphism. Fix $t$ sufficiently small so that this is the case.

In the configuration space we fix a slice around the trivial solution corresponding to the action of the based gauge group, by using the Coulomb gauge fixing condition $d^{*} a=0$. (For any $S^{1}$ connection $A$ on $P$, the imaginary 1-form $a$ is given by $a=A-\theta$, where $\theta$ is the trivial connection.) In this slice we fix a bump function $b(\psi, a)$ which depends smoothly on the $L_{2}^{2}$ norms of $\psi$ and $a$, equal to 1 in a small neighborhood of the trivial solution and equal to 0 outside of a slightly bigger neighborhood. Clearly $b(\psi, a)$ is $J$ invariant.

Now we are ready for a perturbation of the equations. We take the equations $\mathcal{F}_{t}$. Outside of a small neighborhood of the trivial solution they agree with the usual $\not_{A} \psi=0, F_{A}^{+}=q(\psi)$, and inside the neighborhood they are defined on the slice:

$$
\begin{gathered}
\not_{A}(\psi)=t b(\psi, a) P(\Pi(\psi)) \\
F_{A}^{+}=q(\psi)+t b(\psi, a) R\left(\Pi^{\prime}(\psi)\right) .
\end{gathered}
$$

Here $\Pi$ is the $L^{2}$-projection from $\Gamma\left(S^{+}(P)\right)$ to $\operatorname{Ker} \not \partial$, and $\Pi^{\prime}$ is the $L^{2}$ projection from $\Gamma\left(S^{+}(P)\right)$ to $\operatorname{Ker} \not \partial \cap \operatorname{Ker} P$. Since $J$ acts by $L^{2}$-isometries on $\Gamma\left(S^{+}(P)\right)$ and since the kernels are $J$-invariant, these projections are clearly $J$-invariant. 
Now modding out the solution space for $\mathcal{F}_{t}$ by the full gauge-group we get the moduli space $\mathcal{M}_{t}$.

Claim 2.1. If $|t|$ is sufficiently small, then $\mathcal{M}_{t}$ is compact.

Proof. Away from a small ball around $\theta_{0}$ the equations agree with the usual Seiberg-Witten equations and we have compactness by the standard arguments. Inside the ball, by definition, we have bounds on the $L_{2}^{2}$ norms of $a$ and $\psi$ and by standard bootstrapping arguments using the elliptic equations, and the fact that the image of $P$ and $R$ are harmonic, proves that $a$ and $\psi$ are bounded in $L_{3}^{2}$. It follows that we have compactness in this ball as well.

This means that provided only that $t$ is sufficiently small, the above deformation is a legitimate one for computing Seiberg-Witten invariants. Notice that for any $t$ the only reducible solution (up to gauge) to the equations $\mathcal{F}_{t}$ is $\theta_{0}$. Note also that, for generic $t, \theta_{0}$ is isolated in $\mathcal{M}_{t}$ since the quadratic term of the Kuranishi map around this point is given by

$$
Q_{0}+t R: \operatorname{Ker} P \rightarrow \mathcal{H}_{+}^{2}(X ; i \mathbb{R}) .
$$

Now we compute the Seiberg-Witten invariant in two steps. First compute the contribution of $\mathcal{N}_{t}=\mathcal{M}_{t}-\theta_{0}$, which, since $\theta_{0}$ is isolated in $\mathcal{M}_{t}$, is compact. Note that the action of $j$ induces a fix-point free involution on the space $\mathcal{B}^{*}$ of gauge equivalence classes of irreducible configurations and on the subspace $\mathcal{N}_{t}$, and this action preserves the orientation of the determinant line bundle of the elliptic complex corresponding to the perturbed Seiberg-Witten equations $\mathcal{F}_{t}$. The compact space $\mathcal{N}_{t} / j$ is the zeros of a Freedholm section of a Hilbert-bundle over $\mathcal{B}^{*} / j$. This index of this section is zero, though $\mathcal{N}_{t} / j$ may well be of positive dimension. Now we perturb the Freedholm section slightly over $\mathcal{N}_{t} / j$ to make it transverse, and replace $\mathcal{N}_{t} / j$ with a finite set of nondegenerate points. For example if $\mathcal{N}_{t} / j$ were a smooth manifold, the perturbation would be a generic section of the Kuranishi obstruction bundle over $\mathcal{N}_{t} / j$. Details of this sort of arguments can be found for example in Theorem 1.1 of [LT].

Lifting this perturbation to $\mathcal{N}_{t}$ gives a perturbation there, and produces a moduli space with even number of points each being a non-degenerate solution.

Now we use one more perturbation around the trivial solution by adding a small self-dual harmonic two form to the curvature equation in $\mathcal{F}_{t}$. Using a similar bump function as before we can make this perturbation in such a way, that it won't effect $\mathcal{N}_{t}$. Now the contribution of $\theta_{0}$ is equal to the number of points counted with signs in $\left(Q_{0}+t R\right)^{-1}(h) /\left(S^{1}\right)$, where $h$ is a small self-dual harmonic two form, and $S^{1}$ is the stabilizer of $\theta_{0}$ which acts on $\operatorname{Ker} P=\mathbb{C}^{2}$ as complex multiplication. Since $Q_{0}+t R$ is a quadratic form whose associated linear map is an isomorphism, it follows that the contribution of the trivial solution is equal to \pm 1 .

Now we see that this perturbed Seiberg-Witten moduli space contains odd number of non-degenerate solutions. It is also easy to see, that our perturbation can be connected by a one parameter family to a generic perturbation of the 
Seiberg-Witten equations in such a way, that the corresponding one dimensional moduli space doesn't contain reducible solutions. That finishes the proof of Theorem 1.1.

Remark 2.2. Note that Theorem 1.1 can be easily generalized. The same argument shows for instance that for any smooth closed spin four-manifold $X$ with $b_{1}(X)=0, b_{2}^{+}(X)=3, b_{2}^{-}(X)=19$, the Seiberg-Witten invariant of $X$ corresponding to the trivial Spin $^{c}$ structure is congruent to $1 \bmod 2$.

Similarly we can generalize Theorem 1.1 to different intersection forms. The interesting cases are the homotopy types of simply-connected spin elliptic surfaces, since in these cases the formal dimensions of the Seiberg-Witten moduli spaces corresponding to the trivial $\operatorname{Spin}^{c}$ structure are equal to 0 . We can use a similar $J$-invariant perturbation as above to isolate the trivial solution and show that the contribution of the rest of the moduli space is divisible by 2 . The only difference is that if $b_{2}^{+}>3$, then the contribution of the trivial solution is also divisible by 2 . So we get the following result:

Theorem 2.3. Let $X$ be a smooth closed spin manifold with

$$
b_{1}(X)=0, b_{2}^{+}(X)=4 n-1, b_{2}^{-}(X)=20 n-1,
$$

where $n>1$. Then the Seiberg-Witten invariant of $X$ corresponding to the trivial Spin $^{c}$ structure is divisible by 2.

\section{References}

[D1] S. K. Donaldson, Polynomial invariants for smooth 4-manifolds, Topology 29 (1990) $257-315$.

[D2] , The Seiberg-Witten equations and 4-manifold topology, Bull. Amer. Math. Soc. 33 (1996), 45-70.

[FS1] R. Fintushel and R. Stern, Homotopy K3 surfaces containing $\Sigma(2,3,7)$, J. Diff. Geom. 34 (1991), 255-265.

[FS2] - 2-torsion instanton invariants, J. Amer. Math. Soc. 6 (1993), 299-339.

[LT] J. Li and G. Tian, Virtual moduli cycles and Gromov-Witten invariants of general symplectic manifolds, preprint, 1996.

[KM] P. B. Kronheimer and T. S. Mrowka, The genus of embedded surfaces in the projective plane, Math. Res. Lett. 2 (1994), 797-808.

[MSzT] J. W. Morgan, Z. Szabó and C.H. Taubes, A product formula for the Seiberg-Witten invariants and the generalized Thom conjecture, to appear in J. Diff. Geom.

[W] E. Witten, Monopoles and four-manifolds, Math. Res. Letters 1 (1994), 764-796.

Department of Mathematics, Columbia University, New York, NY 10027

E-mail address: jm@math.columbia.edu

Department of Mathematics, Princeton University, Princeton, NJ 08544-1000

E-mail address: szabo@math.princeton.edu 